\title{
Reacciones por lepra en un centro de referencia nacional en Colombia
}

\author{
John Nova, Guillermo Sánchez \\ Centro Dermatológico Federico Lleras Acosta, E.S.E, Bogotá, D.C., Colombia
}

\begin{abstract}
Introducción. Colombia es el país de América con mayor proporción de casos nuevos de lepra con discapacidad grave. Para disminuir tal discapacidad se requiere el control de las reacciones, principal causa del daño neural en esta enfermedad.

Objetivo. Describir las características clínicas y epidemiológicas y el tratamiento de los pacientes con reacciones de tipo 1 y 2 que consultaron al Centro Dermatológico Federico Lleras Acosta.

Materiales y métodos. Se trata de un estudio descriptivo que incluyó la población de pacientes con diagnóstico clínico de reacciones de tipo 1 y de tipo 2 por lepra, que acudieron al centro entre los años 2003 y 2009.

Resultados. Se estudiaron 96 reacciones, 35 del tipo 1 y 61 del tipo 2. El $75 \%$ de los pacientes provenía de los departamentos de Tolima, Cundinamarca, Santander y Boyacá. El 56 \% de las reacciones de tipo 1 se presentaron antes de iniciar la poliquimioterapia para la lepra; el dermatólogo tratante consideró que las reacciones que se presentaron después de suspender la poliquimioterapia eran recaídas. El $94 \%$ de las reacciones de tipo 1 se trataron con corticoides orales. El $97 \%$ de los pacientes con reacciones de tipo 2 presentaron eritema nudoso, $y$ todos se trataron con talidomida.

Conclusiones. La clínica de la reacción de tipo 1 puede orientar al diagnóstico de la lepra en un paciente sin el antecedente de esta enfermedad (56\%). La reacción de tipo 1 que se inicia después de suspender la poliquimioterapia para la lepra, podría ser una manifestación de recaída de la enfermedad. La reacción de tipo 2 es más frecuente en hombres, con una relación hombre a mujer de 4:1. El $97 \%$ de los pacientes con reacción de tipo 2 presentó eritema nudoso.
\end{abstract}

Palabras clave: lepra, eritema nudoso, neuritis, personas con discapacidad.

doi: http://dx.doi.org/10.7705/biomedica.v33i1.582

\section{Leprosy reactions in a Colombian national reference centre}

Introduction: Colombia is the country in America with the highest proportion of new cases leprosy with severe disability. To decrease such disability it is necessary to control these reactions, the main cause of nerve damage in leprosy.

Objective: To describe the clinical and epidemiological characteristics and the treatment of patients with type 1 and 2 leprosy reactions who consulted the Centro Dermatológico Federico Lleras Acosta.

Materials and methods: It is a descriptive study which included patients with clinical diagnoses of type 1 and 2 reactions who were seen in the center between 2003 and 2009. The town of origin of the patients, their age, clinical features and treatments were analysed.

Results: We studied 96 reactions in 87 patients, 35 type 1 and 61 type 2 reactions; $75 \%$ of the patients came from the departments of Tolima, Cundinamarca, Santander and Boyacá; $77 \%$ of type 1 reaction occurred before the beginning of multidrug therapy for leprosy. The reactions that started after stopping the multidrug therapy were considered as a leprosy relapse.

Conclusions: Correct identification of type 1 reaction by the general practitioner will allow the diagnosis of leprosy in a large percentage of patients. The type 1 reaction that begins after stopping the leprosy multidrug therapy may be a manifestation of a relapse of the disease.

Key words: leprosy, erythema nodosum, neuritis, disabled persons.

doi: http://dx.doi.org/10.7705/biomedica.v33i1.582

\footnotetext{
Correspondencia:

John Nova, Avenida 1 No 13a-61, Bogotá, D.C., Colombia.

Teléfono: (571) 242 8160, extensión 199; fax: (571) 2899724

johnnovavilla@yahoo.com
}

Recibido: 25/10/11; aceptado:18/09/12
La lepra, o enfermedad de Hansen, es una enfermedad de la piel y los nervios periféricos causada por el bacilo Mycobacterium leprae (1). Aunque ha disminuido la incidencia de esta enfermedad, no lo ha hecho tanto como se esperaba, a pesar de la introducción de la poliquimioterapia (rifampicina, dapsona y clofazimina) 
por la Organización Mundial de Salud (OMS) hace tres décadas (2-7). Esto ha llevado a que siga siendo un problema de salud pública en países de Asia, África y Suramérica.

Las reacciones en lepra son episodios de inflamación aguda, mediados inmunológicamente, que comprometen la piel y los nervios, principalmente. Las más frecuentes son la reacción de tipo 1, o reacción de reversa, y la reacción de tipo 2 , o eritema nudoso leproso. La reacción de tipo 1 se origina por el aumento de la respuesta inmunitaria mediada por células contra los antígenos de $M$. leprae, con mayor expresión de citocinas del patrón Th1, como la IL-1 $\beta$, TNFa e INF $\gamma(8,9)$. Por otra parte, la de tipo 2 es una reacción inflamatoria sistémica, causada por el depósito de complejos inmunitarios, en la cual predominan el TNFa y las IL6, IL8 y IL10 (8). Las reacciones de tipo 1 y de tipo 2 se pueden presentar hasta en $30 \%$ y $20 \%$ de los pacientes con enfermedad de Hansen, respectivamente $(1,10)$.

Las reacciones son la principal causa de daño neural en la lepra y producen anestesia y deformidades permanentes en las manos y en los pies (11-13). Colombia es el país de América que reporta la mayor proporción de casos nuevos de lepra con discapacidad de grado 2 por secuelas, como dedos en garra, reabsorción de falanges, queratitis, cataratas, ceguera, lesiones perforantes plantares o dedos en martillo (4). Estas secuelas se pueden prevenir con el diagnóstico temprano de la enfermedad y un tratamiento adecuado.

La caracterización de la población colombiana que presenta reacciones por lepra permitirá identificar los aspectos relevantes que pueden contribuir a la implementación de políticas de salud pública en busca de la disminución de la incidencia y prevalencia de las reacciones y sus secuelas y, por ende, un mejor pronóstico. A continuación se describen las características demográficas y clínicas y los tratamientos instaurados en los pacientes con reacción de tipo 1 y de tipo 2 atendidos en un hospital de tercer nivel, de referencia nacional en lepra en Colombia.

\section{Materiales y métodos}

Se desarrolló un estudio observacional y descriptivo. Se incluyeron los pacientes con diagnóstico confirmado de lepra y cuadro clínico de reacción de tipo 1 y de tipo 2, atendidos en el Centro Dermatológico durante los años 2003 a 2009. Se estudiaron variables socio-demográficas y clínicas, y los tratamientos prescritos. Para reportar el índice bacilar obtenido de la baciloscopia, se utilizó la escala colombiana (14). Los datos se extrajeron de las historias clínicas y aquellas con registros incompletos se excluyeron del estudio.

Se definió como reacción de tipo 1 el diagnóstico confirmado nuevo registrado en la historia clínica, basado en manifestaciones clínicas como cambios súbitos en alguna o todas las lesiones, tales como eritema, edema, descamación o aparición de nuevas lesiones o neuritis, es decir, dolor a la palpación en el trayecto superficial del nervio cubital, mediano, ciático poplíteo externo o tibial posterior. Asimismo, se definió como reacción de tipo 2 el diagnóstico confirmado nuevo registrado en la historia clínica, basado en manifestaciones clínicas del paciente como lesiones de eritema nudoso, o fiebre, artralgias, neuritis u orquitis.

Se diagnosticó un nuevo episodio de reacción (de tipo 1 o 2) cuando aparecieron signos o síntomas indicativos después de un periodo asintomático de, por lo menos, seis meses. La procedencia se definió como el departamento donde el paciente vivió antes de diagnosticarse la enfermedad de Hansen y, la residencia, como el departamento donde el paciente vivía al momento del diagnóstico de la reacción.

Se hizo un análisis descriptivo de todas las variables. Se usaron los promedios y las medidas de tendencia central para resumir las variables cualitativas y cuantitativas, respectivamente. Para el análisis se utilizó el programa estadístico Stata$I^{T}{ }^{T M}$, versión 10.0.

\section{Resultados}

\section{Reacción de tipo 1}

Se incluyó información de 33 pacientes que presentaron 35 reacciones de tipo 1. La edad osciló entre 18 y 76 años, con un promedio de 53 años. El $54 \%$ de las reacciones se presentaron en mujeres. El $80 \%$ de los pacientes procedía de los departamentos de Tolima (26\%), Cundinamarca (23\%), Santander (17\%) y Boyacá (14\%). Con respecto a los departamentos de residencia, el $71 \%$ residía en Cundinamarca y, de estos, el 53 $\%$ en Bogotá. Otros departamentos de residencia fueron Tolima (11\%), Santander (6\%), Norte de Santander (6\%), Caldas (3\%) y Boyacá (3\%).

El $34 \%$ de los casos con reacción de tipo 1 eran paucibacilares. El $56 \%$ presentó la reacción de tipo 1 antes de iniciar la poliquimioterapia para la lepra, y el 26 \% desarrolló la reacción dentro 
de los tres primeros meses de tratamiento. Sólo tres presentaron reacción de tipo 1 cuando se encontraban en vigilancia y a estos se les diagnosticó recaída de la enfermedad de Hansen.

El $46 \%$ tenía un índice bacilar de 0 al momento de la reacción de tipo $1 \mathrm{y}$, el $43 \%$, uno menor de 1 . Sólo una paciente con lepra dimorfa tuberculoide tuvo antecedentes de reacción de tipo 2.

Las manifestaciones clínicas se resumen en el cuadro 1. La característica clínica más relevante fue la aparición de placas infiltradas, eritematosas y edematosas (figura 1). Con respecto a los casos de neuritis, el $42 \%$ presentó compromiso de un solo nervio (cubital o mediano). El nervio más comprometido fue el cubital, en $63 \%$ de todos los casos.

En cuanto al tratamiento de la reacción, el $94 \%$ recibió un solo medicamento, principalmente corticoides (91\%). La dosis promedio inicial fue de $30 \mathrm{mg}$ diarios y la dosis máxima utilizada fue de 80 mg diarios. El tiempo de tratamiento varió entre 1 y 74 meses, pero el $50 \%$ recibió el medicamento entre 6 y 18 meses. Todos los pacientes que recibieron corticoides mejoraron en algún momento de la evolución.

Nueve pacientes (29\%) recibieron cloroquina, con dosis que oscilaron entre 250 y $500 \mathrm{mg}$ diarios. Sólo uno de ellos recibió el medicamento como único tratamiento en dos episodios de reacción. El $50 \%$ tomó el medicamento por un lapso de 4 a 20 semanas.

Cinco pacientes recibieron clofazimina a dosis de 300 mg diarios; todos venían recibiendo corticoides orales y uno de ellos cloroquina; cuatro de esos pacientes recibieron el medicamento durante cuatro semanas y uno durante 32 semanas.

El tiempo promedio de duración de la reacción de tipo 1 fue de 14 meses.

Cuadro 1. Características clínicas de los pacientes con reacción de tipo 1

\begin{tabular}{lcc}
\hline Manifestación clínica & $\mathbf{n}$ & (\%) \\
\hline Eritema & 31 & 89 \\
Infiltración & 24 & 69 \\
Aparición de nuevas lesiones & 23 & 66 \\
Neuritis & 19 & 54 \\
Empeoramiento de lesiones preexistentes & 15 & 43 \\
Descamación & 14 & 40 \\
Edema de manos y pies & 9 & 26 \\
\hline
\end{tabular}

$\mathrm{n}=35$ reacciones

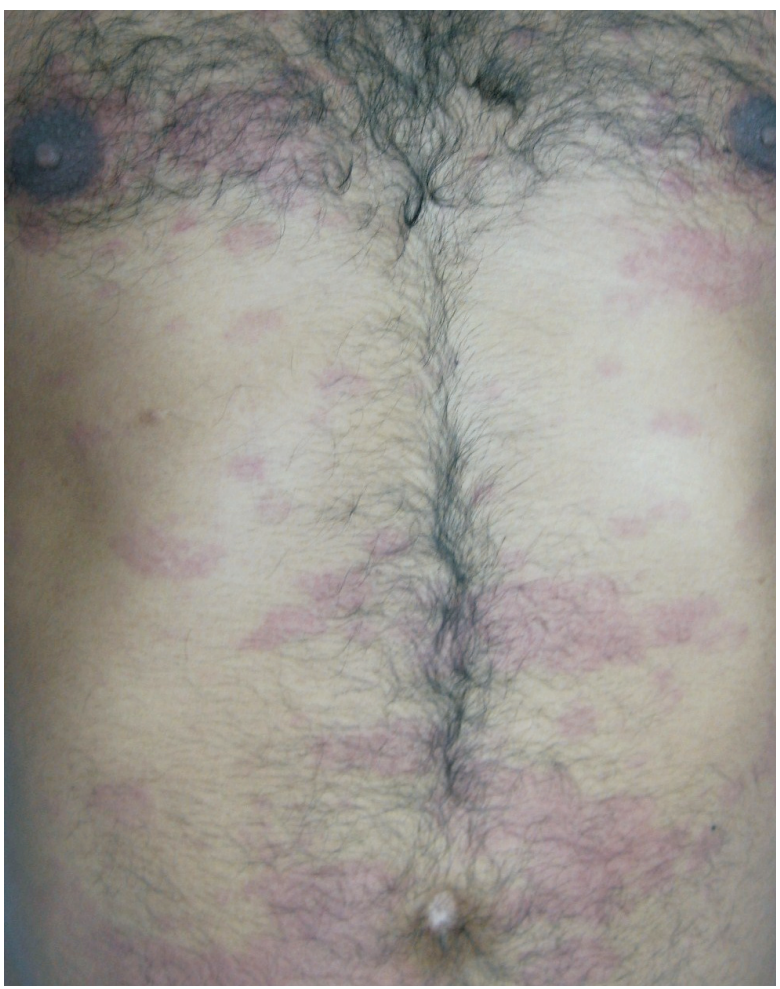

Figura 1. Placas eritemato-edematosas de reciente aparición en un paciente con reacción de tipo 1

En el cuadro 2 se presentan comparativamente los resultados más importantes de las reacciones de tipo 1 y 2 .

\section{Reacción de tipo 2}

Durante el periodo de estudio se incluyeron datos de 58 pacientes, 4 de los cuales tuvieron dos reacciones de tipo 2 durante el seguimiento.

El promedio de edad fue de 44 años, y las edades extremas fueron 15 y 77 años. El $79 \%$ de las reacciones se presentó en hombres.

Aunque el $61 \%$ de los pacientes con reacción de tipo 2 procedía de los departamentos de Cundinamarca, Santander y Boyacá antes del diagnóstico de reacción, algunos venían de departamentos distantes de Bogotá, como Cesar (3,28 \%), Putumayo (1,64 \%) y Chocó (1,64\%). Con respecto a los departamentos de residencia, el 85 \% vivía en Cundinamarca cuando presentaron la reacción y, de estos, el 75 \% vivía en Bogotá.

El promedio del índice bacilar al inicio de la poliquimioterapia fue de 2,3 y, al inicio de la reacción, de 1,9. El $25 \%$ tenía un índice bacilar por encima de 2,5 al inicio de la reacción. 
Cuadro 2. Características diferenciales entre pacientes con reacción de tipo 1 y de tipo 2

\begin{tabular}{llcc}
\hline Característica & & Reacción de tipo $\mathbf{1}$ & Reacción de tipo 2 \\
\hline Promedio de edad (años) & & 53 & 44 \\
& DT & 43 & 0 \\
Tipo de lepra (clasificación de & DD & 34 & 2 \\
Ridley y Jopling) & DL & 9 & 8 \\
& LL & 0 & 90 \\
Momento de presentación & Antes de iniciar el & $56 \%$ & $69 \%$ \\
de la enfermedad & tratamiento para la lepra & $35 \%$ & $3 \%$ \\
& Durante el tratamiento & $9 \%$ & \\
Duración de la reacción (media) (meses) & Después de terminar el & & \\
Duración de la reacción (mediana) (meses) & 14 & 44 & 36 \\
Tratamiento con corticoides & tratamiento & $11 \%$ & $100 \%$ \\
Tratamiento con talidomida & $94 \%$ & & \\
\hline
\end{tabular}

DT: lepra dimorfa tuberculoide; DD: lepra dimorfa dimorfa; DL: lepra dimorfa lepromatosa; LL: lepra lepromatosa

El $28 \%$ de los casos de reacción de tipo 2 se presentó antes del diagnóstico de lepra y, el $69 \%$, durante el tratamiento con varios fármacos. Las manifestaciones clínicas de la reacción de tipo 2 se resumen en el cuadro 3. El $97 \%$ de los casos presentó lesiones de eritema nudoso (figura 2) y sólo uno presentó adenopatías y ulceración de las lesiones.

En tres pacientes se consideró que la reacción de tipo 2 que se inició durante la vigilancia era la manifestación clínica de una recaída de la enfermedad de Hansen, teniendo en cuenta que no tenían antecedentes de reacción. De tal manera, se supuso que se habrían requerido presentación de nuevos antígenos y bacilos viables para iniciar la respuesta inflamatoria de la reacción. En el $20 \%$ el diagnóstico de la reacción se confirmó por biopsia.

Con respecto a los que presentaron neuritis, el $66 \%$ (27 casos) presentó compromiso del nervio cubital, seguido por el del ciático poplíteo externo (12 casos) y el mediano (10 casos). El $48 \%$ presentó compromiso de más de un nervio.

Con respecto al tratamiento, todos los casos se manejaron con talidomida. La dosis inicial promedio, así como la dosis de mantenimiento, fue de $100 \mathrm{mg}$ diarios. El $26 \%$ de los pacientes requirió dosis de 200 mg diarios o más para controlar la reacción.

El tiempo promedio de tratamiento con talidomida fue de 44 meses. El $50 \%$ de los casos requirió entre 15 y 62 meses de tratamiento. El $70 \%$ requirió dos o más años de tratamiento con talidomida. El tiempo requerido más largo fue de nueve años. Todos mejoraron con talidomida, excepto uno, en quien persistió la neuritis a pesar de la resolución de las lesiones de eritema nudoso.
Cuadro 3. Características clínicas de los pacientes con reacción de tipo 2

\begin{tabular}{|c|c|c|}
\hline & Manifestación clínica & $\%(n / 61)$ \\
\hline \multicolumn{2}{|l|}{ Eritema nudoso } & 97 \\
\hline \multicolumn{2}{|l|}{ Neuritis } & 44 \\
\hline \multicolumn{2}{|l|}{ Orquitis-epididimitis } & 18 \\
\hline \multicolumn{2}{|l|}{ Malestar general } & 18 \\
\hline \multicolumn{2}{|l|}{ Edema de manos y pies } & 15 \\
\hline \multicolumn{2}{|l|}{ Fiebre } & 8 \\
\hline \multicolumn{2}{|l|}{ Artritis } & 8 \\
\hline \multicolumn{2}{|l|}{ Conjuntivitis } & 5 \\
\hline \multicolumn{2}{|l|}{ Ulceración lesiones } & 2 \\
\hline Total de reacciones & 61 & \\
\hline
\end{tabular}

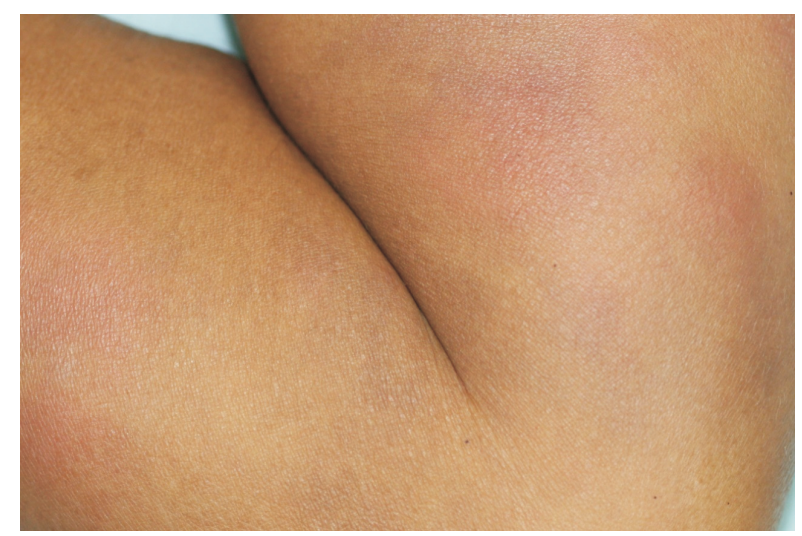

Figura 2. Eritema nudoso leproso en un paciente con reacción de tipo 2

Siete pacientes $(11 \%)$ requirieron corticoides adicionales a la talidomida, con una dosis inicial promedio de $30 \mathrm{mg}$ diarios y una dosis máxima de $35 \mathrm{mg}$ diarios. El tiempo promedio del tratamiento fue de 15 meses. Cinco de los siete pacientes presentaron mejoría clínica cuando se agregó el corticoide. 
Cinco recibieron cloroquina además de la talidomida, tres a dosis inicial de $500 \mathrm{mg}$ diarios y dos a 250 mg diarios; el promedio de duración del tratamiento fue de 10 semanas. Tres de los cinco pacientes refirieron mejoría parcial con la adición de cloroquina.

Cinco recibieron tratamiento con clofazimina, todos a una dosis de $300 \mathrm{mg}$ diarios, cuatro de ellos durante 5 semanas y uno durante 11 meses; este último finalmente se controló solo con talidomida.

El tiempo promedio de duración de la reacción de tipo 2 fue de 44 meses.

\section{Discusión}

Las variables de edad y sexo tuvieron un comportamiento inesperado porque los pacientes con reacción de tipo 2 , en los que predomina una reacción de tipo Th2 (8), fueron, en promedio, una década más jóvenes que aquellos con reacción de tipo 1. Con respecto al sexo, la reacción de tipo 2 predominó en el sexo masculino con una relación hombre a mujer de 3,7:1, a diferencia de la reacción de tipo 1, cuya frecuencia fue similar en hombres y en mujeres. En una cohorte retrospectiva en China, ambas reacciones fueron más frecuentes en hombres que en mujeres, con una razón de 2 a 1, sin diferencias con respecto a la edad (9). En la cohorte de 2.664 pacientes de Bangladesh, la incidencia de daño neural, incluyendo el causado por reacciones, fue de 4,7 para los hombres y de 2,5 para las mujeres (15). Sin embargo, en la cohorte de pacientes etíopes, ambas reacciones fueron un poco más frecuentes en las mujeres $(16,17)$.

Estos trabajos tienen resultados antagónicos a los nuestros, porque en Colombia y en otros países de América predomina la lepra multibacilar $y$, por lo tanto, es más frecuente la reacción de tipo $2(18,19)$, por el contrario, en los países asiáticos predomina la lepra paucibacilar y, por lo tanto, la reacción de tipo 1. En Brasil, el sexo masculino tiene mayor riesgo de desarrollar formas multibacilares (20). Es probable que factores genéticos, ambientales e inmunológicos relacionados con estas poblaciones, determinen el espectro de la lepra y, por lo tanto, el predominio de reacción de tipo 1 o $2(21,22)$.

Para ambas reacciones, los departamentos de donde provenía la mayoría de pacientes (75 \%) fueron Tolima, Santander, Cundinamarca y Boyacá, cercanos a Bogotá, sitio de ubicación del centro de estudio. Es posible que aunque haya casos de lepra en el resto del país, estos sean atendidos en otras regiones y no sean remitidos a Bogotá, teniendo en cuenta que el programa de lepra en Colombia esta descentralizado. Sin embargo, el hallazgo de pacientes procedentes de áreas distantes de Bogotá, como Chocó, Cauca y Putumayo, podría reflejar fallas en los servicios de salud de estas zonas, en cuanto al diagnóstico de la enfermedad. Como se ha documentado por el área de trabajo social del hospital, algunos de estos pacientes fueron remitidos de otras instituciones, otros cambiaron de ciudad para evitar el estigma social y otros, a pesar de haber consultado a su servicio de salud, no habían sido diagnosticados.

Otro hallazgo importante para ambas reacciones, es el cambio de residencia a Cundinamarca una vez se hace el diagnóstico de lepra. Esto es más relevante en nuestra población, teniendo en cuenta que es en Bogotá donde reside el $75 \%$ de los casos una vez están en tratamiento. Los cambios de residencia se explican posiblemente por la necesidad de acudir a controles médicos frecuentes para tratar la reacción. Infortunadamente, no se encontraron registros de las secuelas posteriores a la reacción. Estos resultados plantean la necesidad de contar con un programa nacional y distrital, que permita el manejo integral de las reacciones y que incluya, además del manejo clínico, el abordaje social y psicológico de estos pacientes.

Dos de nuestros pacientes presentaron la reacción cuando tenían un diagnóstico inicial de lepra indeterminada; sin embargo, el seguimiento permitió evidenciar que presentaban formas paucibacilares de la enfermedad. De manera similar a lo que se ha descrito en la literatura científica, la reacción de tipo 1 se presentó en casos de lepra dimorfa (1). De hecho, la lepra dimorfa se considera un factor de riesgo para el desarrollo de reacción de tipo 1 (23); sin embargo, un pequeño número de pacientes con formas polares pueden presentar la reacción (10). Por el contraio, la reacción de tipo 2 se presentó en su gran mayoría (98\%) en casos de lepra lepromatosa.

Uno de los resultados más importantes de este trabajo es que al $56 \%$ de los pacientes con reacción de tipo 1 se les diagnosticó la lepra por las manifestaciones clínicas de la reacción. Este porcentaje es casi el doble que el reportado en la India, donde $31 \%$ presentó reacción de tipo 1 al momento de su primera consulta (10). Esto podría ser secundario a un diagnóstico tardío de la lepra por parte del personal de salud y refuerza la importancia de la capacitación en la identificación de la enfermedad y la reacción, especialmente si 
se tiene en cuenta que la demora en la detección de la enfermedad y, por lo tanto en el inicio del tratamiento, es un factor de riesgo para desarrollar reacciones (24), especialmente la de tipo 1.

Entre las manifestaciones clínicas, además del compromiso cutáneo, es muy importante tener en cuenta la neuritis, que en este trabajo se presentó en cerca de la mitad de los pacientes en ambas reacciones, principalmente con compromiso del nervio cubital. La neuritis puede manifestarse como dolor, sensación de hormigueo o pérdida de la función sensitiva o motora; de allí que se requiera un examen neurológico completo y la palpación de los troncos nerviosos superficiales más afectados por la enfermedad (nervios cubital, mediano, ciático poplíteo externo y peroneo).

Con respecto al compromiso cutáneo de la reacción de tipo 2, el $97 \%$ de los pacientes presentó lesiones de eritema nudoso. Aunque el eritema nudoso puede ser una manifestación de diferentes enfermedades, se debe sospechar enfermedad de Hansen cuando los nódulos son numerosos, recurrentes y se presentan no sólo en miembros inferiores sino también en brazos y tronco (25). A diferencia de la reacción de tipo 1, en la cual el paciente sin antecedentes de enfermedad de Hansen consultaba por la reacción, el $69 \%$ de las reacciones de tipo 2 se presentó durante el tratamiento de la enfermedad. En la práctica clínica hemos encontrado que algunos pacientes han suspendido la poliquimioterapia porque consideran que la reacción es un efecto secundario de los medicamentos. Esto es grave porque sin un control del bacilo se producirá mayor compromiso neural a largo plazo y resistencia del bacilo a los medicamentos; también, en algunos casos refleja una falta de acompañamiento por parte del servicio de salud.

La reacción de tipo 2 puede producir compromiso sistémico dado por uveítis, ceguera, artritis, orquitis, hipogonadismo, esterilidad y ginecomastia (1). En este estudio se encontraron síntomas sistémicos, como fiebre y malestar general, en menos de $20 \%$ de los casos.

A pesar de que no hay criterios claros para establecer el diagnóstico de recaída porque el bacilo no se puede cultivar en medios usuales, se considera que la aparición de nuevas lesiones luego de suspender la poliquimioterapia podría estar relacionada con el desarrollo de una respuesta antigénica frente a un bacilo intacto (26), lo que podría sugerir falla terapéutica o reinfección. En este estudio, se consideró que aquellos pacientes que parecían curados pero presentaban nuevos signos o síntomas indicativos de reacción de tipo 1, presentaban recaída desde el punto de vista clínico, lo que podría corresponder a una falla terapéutica, por persistencia de bacilos viables 0 convivencia con un sujeto con lepra no tratado. Esto refuerza la importancia del seguimiento y vigilancia de los pacientes después de terminada la poliquimioterapia.

Con respecto al tratamiento, aunque los corticoides son el de primera línea sugerido por la OMS (19), existe preocupación por sus efectos secundarios y controversia en cuanto a su efectividad. La heterogeneidad de las definiciones de daño neural en los diferentes estudios impide hacer una adecuada comparación de los resultados. En lo que sí parece haber un acuerdo es en que los corticoides no son útiles en daños neurales de más de seis meses de evolución (26-29) y que, más importante que la dosis inicial del corticoide, es la duración del tratamiento $(30,31)$. En el Centro Dermatológico se utiliza la prednisolona hasta la resolución completa de la reacción, con disminución gradual de la dosis. También, se ha recomendado el uso de medicamentos ahorradores de corticoides; sin embargo, Marlowe reportó que la adición de azatioprina no se había asociado con un mejor pronóstico (32). Por lo tanto, es necesario llevar a cabo estudios para evaluar la efectividad de los corticoides en la resolución de la neuritis aguda y su efecto a largo plazo en la disminución del daño neural permanente.

Otros medicamentos, como la cloroquina, han sido aún menos estudiados que los corticoides (21) y, aunque los dos pacientes de este estudio a quienes se les administró este medicamento mejoraron, se debe tener presente que las reacciones se pueden resolver espontáneamente o por los efectos antiinflamatorios de la poliquimioterapia (21). La clofazimina se ha usado en el tratamiento de las reacciones por su efecto antiinflamatorio; de hecho, se cree que la dosis de $50 \mathrm{mg}$ diarios de la poliquimioterapia protege parcialmente contra el desarrollo de reacción de tipo 2 . Sin embargo, este efecto se pierde después de un año de suspender el tratamiento. La dosis recomendada para la reacción es de $300 \mathrm{mg}$ diarios y la OMS recomienda mantener esta dosis máximo durante un año, por el riesgo de enteropatía (33). Los datos de este estudio no permiten obtener conclusiones con respecto a la efectividad de este medicamento. Además 
de estudiar los corticoides, se necesita investigar nuevas moléculas con menos efectos secundarios.

Todos los pacientes con reacción de tipo 2 fueron manejados con talidomida, con resolución de la reacción en tiempos variables. El tiempo promedio de tratamiento fue de 44 meses, con una duración máxima de 9,7 años (un paciente), mayor al reportado por otros autores (34). En una cohorte retrospectiva de 2.600 casos de lepra, en India, reportaron que el $64 \%$ de los pacientes tuvo episodios recurrentes de eritema nudoso que continuaron hasta ocho años después de haber iniciado el tratamiento; en este mismo trabajo se encontró que la enfermedad lepromatosa, el sexo femenino y los índices bacilares altos, más de 3 en la escala de Ridley y Joplin eran factores de riesgo para reacción de tipo 2 (10). Aunque la mayoría de los pacientes se controló con una dosis de $100 \mathrm{mg}$ diarios de talidomida, $26 \%$ requirió dosis de 200 mg diarios o más.

El uso de la talidomida es limitado en algunos países por su costo y efectos secundarios, especialmente, neurotoxicidad y teratogenicidad (1). Sin embargo, sigue siendo el medicamento de elección para el manejo de la reacción de tipo 2 , por su rápido efecto en la resolución de la reacción (33). El uso de talidomida requiere de educación y seguimiento estricto del paciente, que garantice el uso adecuado de anticoncepción en las mujeres en edad fértil. El uso de corticoides, aunque efectivo en la reacción de tipo 2, es cuestionado por la necesidad de mantener el tratamiento por tiempos prolongados y, por lo tanto, mayor probabilidad de efectos secundarios, lo que ha llevado en algunos casos a la adición de fármacos ahorradores de esteroides, como la azatioprina (35).

Teniendo en cuenta que en este trabajo la reacción de tipo 1 fue el motivo de consulta en 18 pacientes sin diagnóstico previo de lepra, se requiere que el personal de salud conozca no sólo las manifestaciones de la enfermedad, sino de las reacciones, para que se haga una detección temprana de la reacción, de la enfermedad y de las recaídas, favoreciendo un inicio temprano del tratamiento y posiblemente una disminución de las secuelas.

Finalmente, se requieren políticas públicas nacionales y distritales que favorezcan el abordaje integral de estos pacientes, especialmente en Bogotá, ya que esta ciudad recibe gran parte de los pacientes con reacciones.

\section{Conflicto de interés}

Los autores declaramos que no incurrimos en conflicto de interés alguno que pueda afectar los resultados de esta investigación.

\section{Financiación}

Este proyecto fue financiado totalmente por el Centro Dermatológico Federico Lleras Acosta.

\section{Referencias}

1. Walker SL, Lockwood DN. Leprosy. Clin Dermatol. 2007;25:165-72. http://dx.doi.org/10.1016/j.clindermatol. 2006.05.012

2. World Health Organization. Global leprosy situation, 2005. Wkly Epidemiol Rec. 2005;80:289-95.

3. Meima A, Richardus JH, Habbema JD. Trends in leprosy case detection worldwide since 1985. Lepr Rev. 2004;75:1933.

4. World Health Organization. Global leprosy situation. Wkly Epidemiol Rec. 2009;84:333-40.

5. World Health Organization. Leprosy today. Fecha de consulta: 25 de mayo de 2011. Disponible en: http://www. who.int/lep/en/.

6. World Health Organization. Leprosy. Fecha de consulta: 25 de mayo de 2011. Disponible en: http://www.who.int/ mediacentre/factsheets/fs101/en/index.html.

7. Lockwood DN, Suneetha S. Leprosy: Too complex a disease for a simple elimination paradigm. Bull World Health Organ. 2005;83:230-5.

8. Rada E, Aranzazu N, Convit J. Respuesta inmunitaria de la enfermedad de Hansen. Revisión. Investigación Clínica. 2009;50:513-27.

9. Shen J, Liu M, Zhou M, Wengzhong L. Occurrence and management of leprosy reaction in China in 2005. Lepr Rev. 2009;80:164-9.

10. Kumar B, Dogra S, Kaur I. Epidemiological characteristics of leprosy reactions: 15 years experience from north India. Int J Lepr Other Mycobact Dis. 2004;72:125-33.

11. Britton WJ,Lockwood DN. Leprosy. Lancet. 2004;363:120919. http://dx.doi.org/10.1016/S0140-6736(04)15952-7

12. Lienhardt C, Fine PE. Type 1 reaction, neuritis and disability in leprosy. What is the current epidemiological situation? Lepr Rev. 1994;65:9-33.

13. Scollard D, Adams L, Gillis T, Krahenbuhl J, Truman R, Williams D. The continuing challenges of leprosy. Clin Microbiol Rev. 2006;19:338-81. http://dx.doi.org/10.1128/ CMR.19.2.338-381.2006

14. Colorado C, Sánchez G, Guerrero M, León C. Confiabilidad y concordancia de dos escalas de lectura de baciloscopias para clasificación y seguimiento de la terapia multidroga en pacientes con lepra. Biomédica. 2011;31:403-9.

15. Croft R, Nicholls $\mathbf{P}$, Richardus J, Smith $\mathbf{W}$. Incidence rates of acute nerve function impairment in leprosy: A prospective cohort analysis after 24 months (The Bangladesh Acute Nerve Damage Study). Lepr Rev. 2000;71:18-33. 
16. Saunderson P, Gebres S, Byass P. Reversal reactions in the skin lesions of AMFES patients: Incidence and risk factors. Lepr Rev. 2000;71:309-17.

17. Saunderson $\mathbf{P}$, Gebre $\mathbf{S}$, Byass $\mathbf{P}$. ENL reactions in the multibacillary cases of the AMFES cohort in central Ethiopia: Incidence and risk factors. Lepr Rev. 2000;71:318-24.

18. Castiblanco CA. Situación de la lepra en Colombia, 2007. Inf Quinc Epidemiol Nac. 2008;13:129-44.

19. World Health Organization. Regional Office for SouthEast Asia New Delhi. Global strategy for further reducing the leprosy burden and sustaining leprosy control activities 2006-2010. Operational guidelines. Lepr Rev. 2006;77:150.

20. Teixera MA, Silveira VM, Franca ER. Characteristics of leprosy reactions in paucibacillary and multibacillary individuals attended at two reference centers in Recife, Pernambuco. Rev Soc Bras Med Trop. 2010;43:287-92. http://dx.doi.org/10.1590/S0037-86822010000300015

21. Walker SL, Lockwood DN. Leprosy type 1 (reversal) reactions and their management. Lepr Rev. 2008;79:37286.

22. Alter A, Alcais A, Abel L, Schurr E. Leprosy as a genetic model for susceptibility to common infectious diseases. Hum Genet. 2008;123:227-35. http://dx.doi.org/10.1007/ s00439-008-0474-z

23. Ranque B, Nquyen VT, Vu HT, Nquyen TH, Nquyen NB, Pham XK, et al. Age is an important risk factor for onset and sequelae of reversal reactions in Vietnamese patients with leprosy. Clin Infect Dis. 2007;44:33-40. http://dx.doi. org/10.1086/509923

24. Nicholls P, Croft R, Richardus J, Withington S, Smith W. Delay in presentation, an indicator for nerve function status at registration and for treatment outcome: The experience of the Bangladesh Acute Nerve Damage Study cohort. Lepr Rev. 2003;74:349-56.

25. Pfatzgraff R RG. Clinical leprosy. In: Hastings RC, editor. Leprosy. Edinburgh: Churchill Livingstone; 1994. p. 237-90.

26. Kaimal S, Thappa DM. Relapse in leprosy. Indian J Dermatol Venereol Leprol. 2009;75:126-35.
27. Croft R, Nicholls $\mathbf{P}$, Richardus J, Smith W. The treatment of acute nerve function impairment in leprosy: Results from a prospective cohort study in Bangladesh. Lepr Rev. 2000;71:154-68.

28. Saunderson P, Gebre S, Desta K, Byass P, Lockwood D. The pattern of leprosy-related neuropathy in the AMFES patients in Ethiopia: Definitions, incidence, risk factors and outcome. Lepr Rev. 2000;71:285-308.

29. van Veen $\mathbf{N}$, Nicholls $\mathbf{P}$, Smith W, Richardus J. Corticosteroids for treating nerve damage in leprosy. A Cochrane Review. Lepr Rev. 2008;79:361-71.

30. Garbino JA, Virmond MCL, Ura S, Salgado MH, Naafs B. A randomized clinical trial of oral steroids for ulnar neuropathy in type 1 and type 2 leprosy reactions. Arq Neuropsiquiatr. 2008;66:861-7. http://dx.doi.org/10.1590/ S0004-282X2008000600016

31. Rao P, Sugamaran D, Richard J, Smith W. Multi-centre, double blind, randomized trial of three steroid regimens in the treatment of type-1 reactions in leprosy. Lepr Rev. 2006;77:25-33.

32. Marlowe SN, Hawksworth RA, Butlin CR, Nicholls PG, Lockwood DN. Clinical outcomes in a randomized controlled study comparing azathioprine and prednisolone versus prednisolone alone in the treatment of severe leprosy type 1 reactions in Nepal. Trans R Soc Trop Med Hyg. 2004;98:602-9. http://dx.doi.org/10.1016/j. trstmh.2003.12.013

33. Walker S, Waters M, Lockwood D. The role of thalidomide in the management of erythema nodosum leprosum. Lepr Rev. 2007;78:197-215.

34. Balagon MV, Gelber RH, Abalos RM, Cellona RV. Reactions following completion of 1 and 2 year multidrug therapy (MDT). Am J Trop Med Hyg. 2010;83:637-44. http:// dx.doi.org/10.4269/ajtmh.2010.09-0586

35. Duraes SM, Salles Sde A, Leite VR, Gazzeta MO. Azathioprine as a steroid sparing agent in leprosy type 2 reacions: Report of nine cases. Lepr Rev. 2011;82:304-9. 